Z. Klin. Chem. Klin. Biochem.

12. Jg. 1974, S. $207-209$

\title{
Zur statistischen Verteilung von IgA, IgG und IgM bei 20-30 jährigen weiblichen und männlichen Normalpersonen
}

Von U. H. Cegla

\begin{abstract}
Aus dem Zentrum der Inneren Medizin, Abteilung für Pneumologie, der Johann Wolfgang Goethe-Universität Frankfurt/M.
\end{abstract}

(Eingegangen am 21. Februar/9. November 1973)

Mit Hilfe von Wahrscheinlichkeitspapier sowie einem Computerprogramm wird der Verteilungstyp (linear oder logarithmisch) der Immunglobuline IgG, IgA und IgM untersucht; IgG wird als linear, dagegen IgA und IgM als logarithmisch verteilt gefunden. Die entsprechenden Mittelwerte und Standardabweichungen werden angegeben und die Befunde im Rahmen der Literatur besprochen.

\section{The statistical distribution of IgA, IgG and IgM in 20-30 year old normal male and female persons}

IgG, IgA and IgM was studied with the aid of a probability network and a computer program. IgG was found to have a linear distribution, whereas IgA and IgM were distributed logarithmically. The corresponding average values and standard deviations are given, and the findings are discussed in relation to the existing literature.

Die Bestimmung der Immunglobuline IgA, IgG und IgM ist heute eine Routinemethode geworden; jedoch sind Mitteilungen über den statistischen Verteilungstyp der Normalwerte nicht sehr zahlreich, als Übersicht sei die Arbeit von Kalff genannt (1).

Es wurden deshalb der Verteilungstyp (linear bzw. logarithmisch), die Mittelwerte sowie die Standardabweichung des Immunglobulins A bei 97 Personen (37 Frauen, 60 Männer) des Immunglobulins $G$ bei 103 Personen (43 Frauen, 63 Männer) sowie des Immunglobulins M bei 111 Personen (43 Frauen, 68 Männer) bestimmt.

Beim Immunglobulin $\mathrm{G}$ und Immunglobulin A konnten wir in Übereinstimmung mit den Arbeiten von Kalff keine geschlechtsbedingten Unterschiede feststellen, so daß diese Immunglobuline zusammen (Frauen und Männer) betrachtet werden (1). Beim Immunglobulin M fanden sich, wie schon von Butterworth et al (2) und Kohler und Farr (3) früher beschrieben, geschlechtsbedingte Unterschiede (Männer hatten niedrigere Spiegel als Frauen), weshalb das IgM im folgenden für beide Geschlechter getrennt betrachtet wird.

\section{Material und Methoden}

Als Normalpersonen wurden solche Probanden (Klinikpersonal und Medizinstudenten) angesehen, bei denen $\mathrm{z}$. Zt. der Untersuchung weder Beschwerden noch Krankheitssymptome bestanden und die auch sonst an keiner chronischen Erkrankung litten. Die Immunglobuline wurden mit der Radioimmunodiffusionsmethode nach Mancini (4) mit Hilfe von Partigenplatten und den entsprechenden Immunglobulinstandardseren der Behringwerke ${ }^{1}$ ) bestimmt.

1) Hersteller: Behringwerke, D.3550 Marburg/Lahn
Die Bestimmung des Verteilungstyps (linear bzw. logarithmisch) erfolgte graphisch mit Hilfe von Wahrscheinlichkeitsnetzen ${ }^{2}$ ). Außer dieser graphischen Methode erfolgte die Bestimmung des Verteilungstyps auf einer Rechenanlage (Typ pdp 11-20 $0^{3}$ ); für 19 verschiedene Funktionen wurde jeweils die kumulative Häufigkeit berechnet und an Hand der Meßwerte die vorkommende Häufigkeit gezählt. Die Funktion mit dem günstigsten (hypothetisch ist ein Korrelationskoeffizient von 1 optimal) Korrelationskoeffizienten zwischen gezählter und gerechneter kumulativer Häufigkeit galt als optimale Funktion.

\section{Ergebnisse}

Abbildung 1 zeigt im linearen Wahrscheinlichkeitsnetz die Häufigkeitssummen von IgA, IgG und IgM. Im einzelnen ergibt sich folgendes:

\section{$\operatorname{Ig} A$}

Es findet sich im linearen Wahrscheinlichkeitsnetz eine nach oben konvexbogige Kurve; es besteht also keine lineare Verteilung des Kollektivs, denn in diesem Falle hätten alle Häufigkeitssummenpunkte auf einer geraden Linie liegen müssen. Im logarithmischen Wahrscheinlichkeitsnetz dagegen liegen fast alle Punkte auf einer Geraden (Abb. 2). Auch bei der Berechnung der Verteilung findet sich eine logarithmische Verteilung des IgA mit einem Mittelwert von

$$
\begin{aligned}
& \overline{\mathrm{x}}=114,48 \text { I.E. } \mathrm{IgA} / \mathrm{ml}+56,42 \text { I.E. } \mathrm{IgA} / \mathrm{ml} \\
& -52,39 \text { I.E. IgA } / \mathrm{ml} \\
& \text { entsprechend } \left.1924,0 \mathrm{mg} \operatorname{IgA} / 1^{4}\right)+948,2 \mathrm{mg} \operatorname{IgA} / 1 \\
& -880,5 \mathrm{mg} \operatorname{IgA} / 1
\end{aligned}
$$

\footnotetext{
2) Wahrschcinlichkeitspapier Nr. 298 1/2 A und 297 1/2 A 3. Herstcller: Carl Schleicher \& Schüll, D 3352 Einbeck

3) Fa. Digital Equipment, 8000 München, Wallenstcinplatz 20
} 


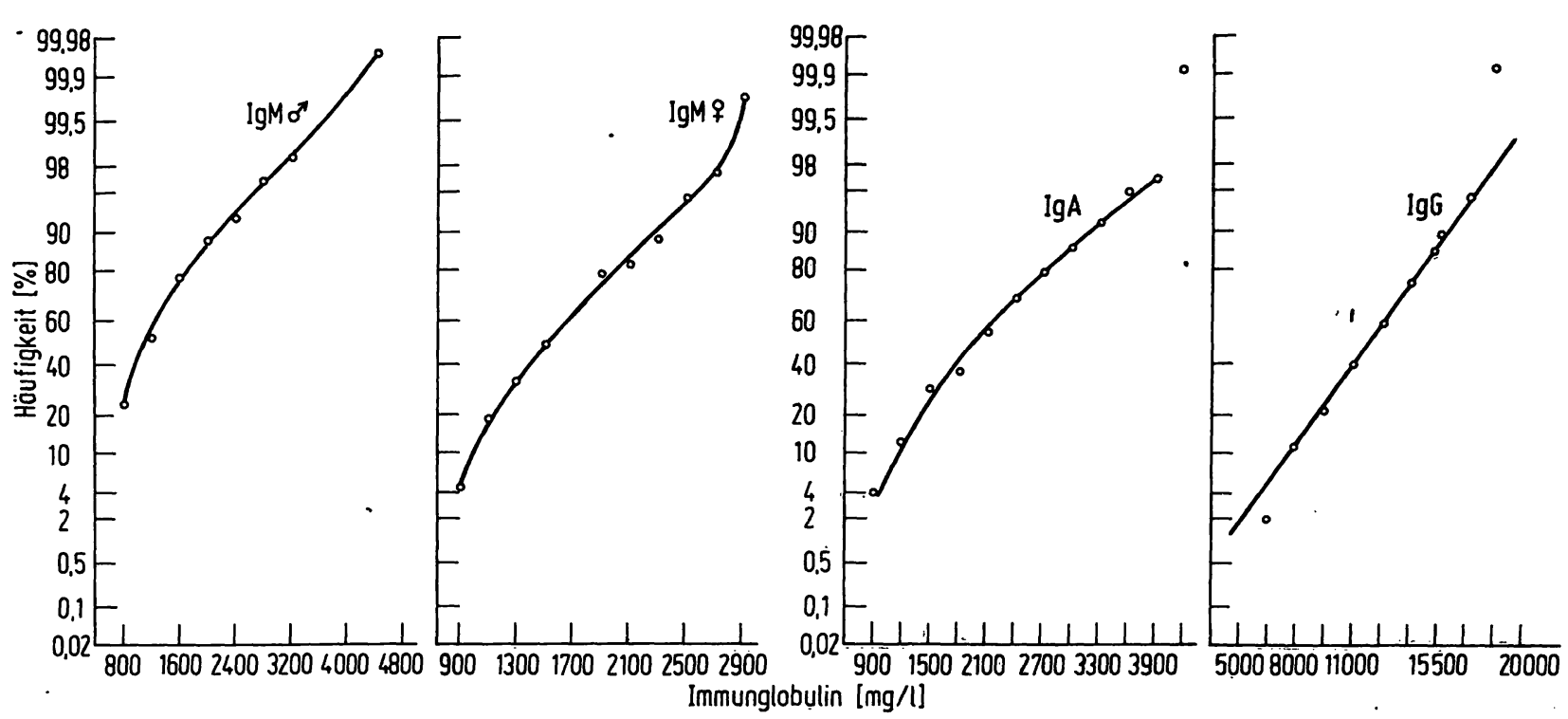

Abb. 1. Summenhäufigkeiten von IgG, IgA und IgM im linearen Wahrscheinlichkeitsnetz (IgM nach Männern und Frauen aufgeteilt).

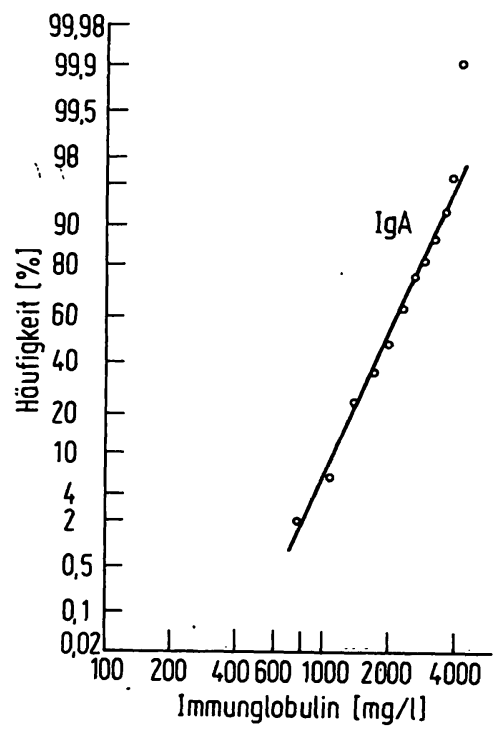

Abb. 2. Summenhäufigkeiten von IgA im logarithmischen Wahrscheinlichkeitsnetz.

\section{$\operatorname{Ig} G$}

Im linearen Wahrscheinlichkeitsnetz (Abb. 1) stellen sich die Häufigkeitssummen als Gerade mit Abweichungen an den Endpunkten dar, was für eine lineare Verteilung eines nicht ganz homogenen Kollektivs spricht. Die Computerberechnung bestätigt eine lineare Verteilung der IgGWerte mit einem Mittelwert von

$\overline{\mathrm{x}}=136,75$ I.E. $\mathrm{IgG} / \mathrm{ml} \pm 33,70$ I.E. $\mathrm{IgG} / \mathrm{ml}$ entsprechend $11891,4 \mathrm{mg} \operatorname{IgG} / 1 \pm 2930,6 \mathrm{mg} \mathrm{IgG/} / 1^{4}$ ) IgM

Die Häufigkeitssummen von IgM im linearen Wahrscheinlichkeitsnetz für Männer und Frauen getrennt aufgetragen zeigen ebenso wie die des IgA einen nach oben konvexbogigen Verlauf und weisen damit auf eine nicht lineare Verteilung hin (Abb. 1). Die Häufigkeitssummen der männlichen Normalpersonen (Abb. 3) im logarithmischen Wahrscheinlichkeitsnetz stellen eine Gerade dar; es handelt sich also um ein logarithmisch verteiltes Kollektiv.

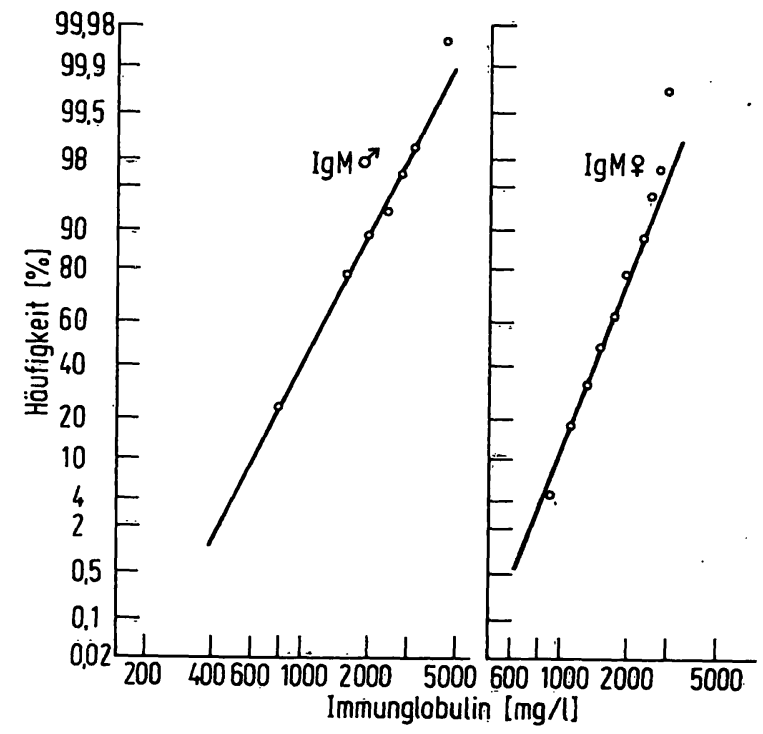

Abb. 3. Summenhäufigkeiten von IgM (Männer und Frauen getrennt) im logarithmischen Wahrscheinlichkeitsnetz.

Die Häufigkeitssummen der weiblichen Personen liegen im logarithmischen Wahrscheinlichkeitșpapier ebenfalls auf einer Geraden (Abb. 3).

Auch beim IgM wird die zeichnerisch gefundene lognormale Verteilung von der Computerberechnung bestätigt; für Frauen berechnete sich:

$$
\begin{array}{r}
\overline{\mathrm{x}}=173,42 \text { I.E. IgM/ml }+62,84 \text { I.E. IgM/ml } \\
-46,12 \text { I.E. IgM/ml } \\
\text { entsprechend } \left.1508,0 \mathrm{mg} \operatorname{IgM} / 1^{4}\right)+546,4 \mathrm{mg} \text { IgM/1 } \\
-401,0 \mathrm{mg} \operatorname{IgM} / 1
\end{array}
$$

Für die Männer berechnete sich der Mittelwert des IgM:

$\overline{\mathrm{x}}=133,11$ I.E. IgM/ml + 80,28 I.E. IgM/ml

$$
-50,07 \text { I.E. IgM/ml }
$$

entsprechend $\left.1157,5 \mathrm{mg} \operatorname{IgM} / 1^{4}\right)+698,1 \mathrm{mg} \mathrm{IgM} / 1$

$$
-435,4 \mathrm{mg} \operatorname{IgM} / 1
$$

4) bezogen auf den IgA-bzw. IgG- und IgM-Standard der Behring-
werke Marburg. 


\section{Diskussion}

Die meisten Veröffentlichungen, die über Immunglobuline berichtet haben, setzen eine lineare Normalverteilung voraus, da sie den Mittelwert mit gleicher Standardabweichung nach oben und unten angeben; in einer Ubersichtsarbeit von Kalff (1) setzen von 31 Autoren 29 eine Normalverteilung voraus.

Allansmith et al (5) beschreiben unseres Wissens als erste eine logarithmische Verteilung der Immunglobuline IgG, IgA und IgM. Ferner berichten Cwynarske (6), Claman et al (7) sowie Goodman und Hobbs (8) und Lichtman et al (9) über eine Nichtnormalverteilung der Immunglobuline.

Im Gegensatz zu Allansmith et al (5) sowie Geiger und Hoffmann (10) fanden wir für IgG eine lineare Verteilung, was den Untersuchungen von Kalff (1) entspricht. Die von uns gefundenen Mittelwerte des IgG stimmen mit denen der o. g. Autoren gut überein.
Die außerordentlich hohe Standardabweichung des IgA von $+189 \%$ und - $64 \%$ des Mittelwertes bei Allansmith et al (5) dürfte durch das Vorliegen einer Mischverteilung bedingt sein.

Der von uns angegebene Mittelwert für IgM stimmt mit den von den Behring-Werken (11) angegebenen Normalwerten gut überein.

Die teleologische Bedeutung des unterschiedlichen Verteilungsmusters der Immunglobuline ist unklar.

\section{Danksagung}

Herrn cand. med. E. Hossenfelder bin ich für die Programmierung zu Dank verpflichtet.

Herrn Prof. Dr. med. W. A. Mondorf und Herrn Dr. rer. nat. M. Kollmar danke ich für das Überlassen von Meßergebnissen sowie für Ratschläge und Anregungen.

\section{Literaturverzeichnis}

1. Kalff, M. W. (1970), Clin. Chim. Acta 28, 277-289

2. Butterworth, M., Mc Clellan, B. \& Allansmith, M. (1967), Nature 214, 1224-1225

3. Kohler, P. F. \& Farr, R. S. (1967), J. Allergy 39, 311-322

4. Mancini, J. G., Vaermann, P., Carbonara, A. O. \& Heremans, J. F. (1964); Protides of the Biological Fluids. Proc. $\mathrm{Xi}^{\text {th }}$ coll. Brügge/Belgien 1963, Elsevier, Amsterdam.

5. Allansmith, M., Mc Clellan, B., \& Buttersworth, M. (1967). Proc. Soc. Exp. Biol. 125, 404-407

6. Cwynarski, M. T. (1968), Clin. Chim. Acta 19, 1-5
7. Claman, H. N. \& Merril, D. (1964), J. Lab. Clin. Med. $64,685-693$

8. Goldman, J. M., \& Hobbs, J. R. (1967), Immunology 13, 421-431

9. Lichtman, M. A., Vaughan, J. H., \& Hames, C. G. (1967) Arthritis Rheum. 10, 204-215

10. Geiger, H. \& Hoffmann, P. (1970), Z. Kinderheilk. 109, 22-40

11. Farbwerke Hoechst AG, Bereich Behring, D. div. 631-649 (11827)

Anschrift des Verfassers:

Dr. med. U. H. Cegla

Abteilung für Pneumologie d. Zentrums d. Inneren Medizin

i. J. W. Goethe-Universität

6000 Frankfurt/M.

Theodor-Stern-Kai 7 